\title{
O impacto das práticas de gestão do conhecimento no desempenho organizacional: um estudo em empresas de base tecnológica
}

Fabricio Ziviani ${ }^{I}$

Erik Paixão Amarante ${ }^{I I}$

Renata de Souza França ${ }^{I I I}$

Paulo Isnard ${ }^{I V}$

Eric de Paula Ferreirav

${ }^{I}$ Universidade Fumec, Belo Horizonte, MG, Brasil.

Doutor em Ciência da Informação pela Universidade Federal de Minas Gerais.

Professor do Programa de Pós-Graduação em Sistemas de Informação e Gestão do Conhecimento da Universidade FUMEC. Docente da Universidade do Estado de Minas Gerais (UEMG). Professor da Fundação Dom Cabral (FDC)

II Mestre em Sistemas de Informação e Gestão do Conhecimento pela Universidade FUMEC.

III Doutoranda em Sistemas de Informação e Gestão do Conhecimento pela Universidade FUMEC.

${ }^{I V}$ Doutorando em Sistemas de Informação e Gestão do Conhecimento pela Universidade FUMEC.

${ }^{v}$ Doutorando em Sistemas de Informação e Gestão do Conhecimento pela Universidade FUMEC.

http://dx.doi.org/10.1590/1981-5344/3468

Para que a gestão do conhecimento impacte os rumos da empresa com inovações e vantagens competitivas, é necessário integrá-la à estratégia organizacional. Apesar de existirem estudos que apontam a correlação entre gestão conhecimento, desempenho e estratégia organizacionais, a prática se torna divergente. As organizações estão cientes da importância da gestão do conhecimento, mas é preciso sair do campo do discurso e converter ações em resultados. O presente trabalho tem como objetivo avaliar o impacto das práticas de gestão do 
conhecimento no desempenho organizacional, através de uma pesquisa quantitativa com a aplicação de um questionário survey em empresas de base tecnológica. A pesquisa utilizou o modelo empírico de Wu e Chen, para a validação das hipóteses definidas no estudo e a técnica de modelagem de equações estruturais foi aplicada. Os resultados obtidos possibilitam confirmar as relações existentes entre as cinco dimensões analisadas- ativos do conhecimento, aprendizagem organizacional, capacidade do processo de conhecimento, capacidade do processo de negócio e desempenho organizacional-, além de identificar que dentre os construtos analisados, 0 processo de negócio foi o que apresentou maior influência sobre o desempenho organizacional.

Palavras-Chave: Conhecimento, Gestão do conhecimento, Desempenho organizacional, Empresas de base tecnológica.

\section{The impact of knowledge management practices on organizational performance: a study on technology- based companies}

In order for knowledge management to impact the company's directions, bringing innovations and competitive advantages, it is necessary to integrate it into the organizational strategy. Although there are studies that point out the correlation between knowledge management, organizational performance and strategy, this often does not occur in practice. Organizations are aware of the importance of knowledge management, but we must get out of the field of discourse and convert actions into results. The present work aims to evaluate the impact of knowledge management practices on organizational performance through a quantitative research with the application of a survey questionnaire in technology - based companies. The research, quantitative denominated,used the empirical model of Wu and Chen, for the validation of the hypotheses defined in the study, the modeling technique of structural equations was applied. The obtained results make it possible to confirm the existing relationships between the five analyzed dimensions- knowledge active, organization learning, 
knowledge process capacity, business management capacity and organizational performance- besides identifying that among the constructs analyzed, the business process was the one that had the greatest influence on the organizational performance.

Keywords: Knowledge, Knowledge Management, Organizational Performance, high-tech enterprises.

Recebido em 08.03.2018 Aceito em 05.09.2018

\section{Introdução}

Para que a gestão do conhecimento impacte os rumos da empresa com inovações e vantagens competitivas, é necessário integrá-la à estratégia organizacional. Para Davenport (1999) o conhecimento é o bem mais valioso da organização, e consequentemente, o mais difícil de gerenciar. A gestão do conhecimento se apresenta como um modelo com um conjunto de técnicas e ferramentas que se ocupam dos processos gerenciais e infraestrutura física e digital que facilitam, favorecem e estimulam os processos humanos de criação, compartilhamento e disseminação de conhecimentos individuais e coletivos (TERRA, 2005).

Para que a gestão do conhecimento cause impactos relevantes na organização é necessário integrá-la à sua estratégia, o que pode causar uma melhora significativa no desempenho da organização. Slater, Olson e Hult (2010) definem desempenho como o atingir dos objetivos estabelecidos pela organização ou a superação destes, podendo ser avaliado por aspectos financeiros, de mercado e/ou inovação.

Para Davenport (1999) poucas empresas têm sido capazes de estabelecer uma relação causal entre as atividades de gestão do conhecimento e o desempenho da organização. Os desafios relacionados à adoção das práticas e modelos associados à Gestão do Conhecimento não são triviais, pois, denotam significativos esforços de conscientização e de comunicação e ativa participação pessoal da alta administração.

No Brasil, existem poucas pesquisas correlacionando de forma prática e linear, gestão do conhecimento e desempenho organizacional. Assim, esta pesquisa propõe-se a oferecer uma contribuição tanto teórica, dissertando sobre os temas abordados, como prática, relacionando a gestão do conhecimento e o desempenho organizacional, por meio da aplicação do modelo teórico empírico de Wu e Chen (2014), em empresas de base tecnológica, de modo a verificar esta relação.

O estudo está distribuído em seis sessões. A primeira sessão trabalha a introdução do assunto. Seguida da segunda sessão, é apresentada uma revisão sistemática explicando as formas de pesquisa para levantamento dos trabalhos e assuntos já existentes. A terceira sessão, trata do referencial teórico explicitando os principais conceitos para entendimento da pesquisa. A quarta sessão apresenta o processo 
metodológico utilizado na pesquisa para atingir os objetivos da pesquisa. Posteriormente, são apresentados os resultados e análises na quinta sessão, bem como as considerações finais na sexta sessão. Por final, são apresentadas as referências bibliográficas utilizadas nessa pesquisa.

\section{Revisão sistemática e apresentação dos trabalhos relacionados à pesquisa}

A revisão sistemática de literatura é uma forma de analisar e interpretar pesquisas relevantes para uma determinada questão, área temática ou de interesse (KITCHENHAM, 2004). Um dos objetivos da revisão sistemática é identificar os trabalhos que relacionam de forma a encontrar uma linearidade entre os construtos.

Foi definida a estratégia da revisão sistemática de literatura em uma consulta geral nas bases de dados, que tem como objetivo detectar de forma relevante o que foi escrito sobre o assunto pesquisado, de acordo com o Quadro 1. Para os descritores Gestão do Conhecimento e Desempenho Organizacional utilizou-se as bases Scientific Periodicals Electronic Library (SPELL), Scientific Eletronic Library Online (Scielo) e Associação Nacional de Pós-Graduação e Pesquisa em Administração (Anpad). Para o descritor Modelos de Gestão do Conhecimento e alguns quesitos de Desempenho Organizacional utilizou-se as bases Emerald Insight, Elseviver, Doiserbia e Canadian Center of Science and Education (CCSE). A escolha se deu devido a representatividade internacional que essas bases possuem em relação aos assuntos envolvidos nessa pesquisa.

QUADRO 1: Atributos da Qualidade da Informação

\begin{tabular}{|l|l|l|}
\hline \multicolumn{1}{|c|}{ DESCRITORES } & \multicolumn{1}{|c|}{ BASE DE DADOS } & \multicolumn{1}{c|}{ SITES } \\
\hline Gestão de conhecimento & SPELL, Scielo, ENANPAD & $\begin{array}{l}\text { http://www.spell.org.br/ } \\
\text { http://www.scielo.org/ } \\
\text { http://www.anpad.org.br/ }\end{array}$ \\
\hline Desempenho organizacional & SPELL, Scielo, ENANPAD & $\begin{array}{l}\text { http://www.spell.org.br/ } \\
\text { http://www.scielo.org/ } \\
\text { http://www.anpad.org.br/ }\end{array}$ \\
\hline $\begin{array}{l}\text { Modelo de gestão do } \\
\text { conhecimento e desempenho } \\
\text { organizacional }\end{array}$ & $\begin{array}{l}\text { Emerald Insight, Elseviver, } \\
\text { http://www.emeraldinsight.com } \\
\text { http://www.elsevier.com/ } \\
\text { http://www.doiserbia.nb.rs/ } \\
\text { http://www.ccsenet.org }\end{array}$ \\
\hline
\end{tabular}

Fonte: Dados da Pesquisa

Definidos os descritores, bem como as bases de referência para pesquisa, o planejamento foi definido em três etapas:

- Pesquisa geral utilizando os descritores relacionadas no Quadro 1;

- Pesquisa estruturada usando os descritores Gestão do Conhecimento, Gestão de Conhecimento e Desempenho organizacional levando em consideração os resultados encontrados nos títulos e resumos dos trabalho; 
- Análise e classificação dos resultados em 3 níveis: Dentro do contexto, a analisar, fora do contexto.

Para a pesquisa, utilizou-se a combinação das palavras chave "Gestão do Conhecimento" e "Desempenho Organizacional". Os resultados obtidos foram usados como referência para a construção do trabalho de pesquisa, com as devidas análises de acordo com os objetivos propostos (KITCHENHAM, 2004).

A partir do levantamento, foram destacados os estudos dos seguintes autores:

- Lee e Choi (2003): Propuseram uma visão integrada do conhecimento gestão e forneceram orientações estratégicas;

- Mills e Smith (2011): Avaliaram os impactos dos recursos de conhecimento específico de gestão sobre o desempenho organizacional;

- Zack et al (2009): Apresentaram os resultados de uma investigação exploratória do impacto da gestão do conhecimento no desempenho organizacional;

- Fugate et al (2009): Destacaram o papel das operações de logística na criação de valor;

- Wu e Chen (2014): Definiram um modelo para avaliar os impactos da gestão do conhecimento sobre o desempenho organizacional.

Por meio dos resultados obtidos na revisão sistemática, pode se identificar vários modelos que relacionam gestão do conhecimento e desempenho organizacional.

\section{Referencial teórico}

Nessa seção é apresentada a sustentação teórica da pesquisa, que examina o valor do conhecimento nas organizações, a gestão do conhecimento, as práticas de gestão do conhecimento, o desempenho organizacional e modelos empíricos que relacionam gestão do conhecimento e desempenho organizacional.

\section{O Valor do conhecimento nas organizações e a Gestão do Conhecimento}

O conhecimento é considerado por Porter (1998) a única vantagem a oferecer diferenciação para uma competição sustentável, ou seja, as empresas devem promover a criação do conhecimento, as melhores práticas e a transferência das competências para toda a organização com o objetivo de aprimorar negócios.

Davenport (1999) reconhece que os aspectos intangíveis que adicionam valor aos produtos e serviços são todos baseados em 
conhecimento. Ao contrário de ativos materiais que se depreciam à medida que são utilizados, o ativo do conhecimento é ilimitado e cresce quando é estimulado e utilizado. Os recursos físicos e materiais não são mais o principal pilar para um desempenho organizacional satisfatório. 0 tamanho das instalações industriais e dos edifícios administrativos de uma empresa deixou de ser uma medida confiável de sua importância ou capacidade industrial, pois, o que agrega valor, inova e faz a diferença é o conhecimento.

O conhecimento só se torna um ativo corporativo quando é acessível, gerenciado e cercado do mesmo cuidado dedicado à obtenção de valor de outros ativos tangíveis (DAVENPORT; PRUSAK, 1999). A gestão do conhecimento surgiu como um importante campo para a prática e pesquisa em sistemas de informação. Este campo foi construído sobre bases teóricas da economia da informação, gestão estratégica, cultura organizacional, comportamento organizacional, estrutura organizacional, inteligência artificial, gestão da qualidade e medição de desempenho organizacional (BASKERVILLE; DULIPOVICI, 2006). A ideia de Dulipovici (2006) é confirmada por Kuriakose et al. (2010), que define a gestão do conhecimento como um campo interdisciplinar que abrange áreas como as tecnologias da informação e comunicação, os sistemas de informação e a gestão de mudanças no intuito de melhorar processos e o desempenho organizacional.

Sabbag (2007) define a gestão do conhecimento nas organizações como um sistema integrado que visa desenvolver conhecimento e competência coletiva para ampliar o capital intelectual e a sabedoria das pessoas. Já para Davenport e Prusak (1999), a gestão do conhecimento refere-se à reunião de todas as tarefas que envolvam a geração, codificação e transferência do conhecimento.

A essência da gestão do conhecimento está na disposição das pessoas para compartilhar suas experiências e para entender o que as pessoas sabem, é preciso reproduzir o contexto dos seus conhecimentos. A gestão do conhecimento está centrada em três aspectos principais: (i) foco nos ativos intangíveis, (ii) tornar a gestão do conhecimento algo explícito e incentivar e (iii) criar mecanismos que facilitem aos empregados o compartilhamento de seus conhecimentos (NONAKA; TAKEUCHI, 1997).

O desafio das empresas está em converter o conhecimento tácito dos funcionários em conhecimento explícito, e para isso Nonaka e Takeuchi (1997) propõem um modelo de conversão do conhecimento. Esse modelo engloba quatro modos de conversão do conhecimento explicados em função das possibilidades de interações entre o conhecimento tácito (constituinte na mente das pessoas) e o explícito (já documentados), sendo a socialização, combinação, internalização e externalização.

A socialização é composta pela interação do conhecimento tácito entre pessoas - conversão de conhecimento tácito em conhecimento tácito; A combinação é o conjunto de conhecimento explícito controlado 
pelas pessoas; A internalização se concentra na conversão de conhecimento explícito em conhecimento tácito; Por fim, a externalização é a conversão de conhecimento tácito em conhecimento explícito.

Nonaka e Takeuchi (1997) afirmam que os quatro modos de conversão do conhecimento devem ser gerenciados de forma articulada e cíclica a fim de dominar o conjunto dos processos que fomentam um movimento cíclico de construção do conhecimento denominado "espiral de criação de conhecimento" ou modelo SECI.

Por meio das propostas explicitadas, percebe-se que a base do conhecimento organizacional consiste em ativos de conhecimento individuais e coletivos que a organização pode utilizar para realizar suas tarefas, bem como nos dados e informações sobre os quais constrói tal conhecimento (PROBST; RAUB; ROMHARDT, 2002).

\section{Práticas de gestão do conhecimento}

As práticas de gestão do conhecimento são conhecidas como rotinas diretamente envolvidas no desenvolvimento e na aplicação do conhecimento. As práticas ajudam as empresas a tomarem medidas orientadas a melhorar as capacidades de resposta e a superarem as limitações da inovação causadas pela cultura e história (COOMBS; HULL; PELTU, 2000). A APO (2009) considera que todas as práticas são fundamentais para as iniciativas de gestão do conhecimento, pois, promovem crescimento e evolução da internalização do capital intelectual nas organizações.

Segundo Becerra-Fernandez e Sabherwal (2001) existem dezenove práticas de gestão do conhecimento para avaliar a maneira como a gestão influi no âmbito organizacional, como mostra o Quadro 2. Retomando o modelo SECI de Nonaka e Takeuchi (1997), buscou se indicar como as práticas de gestão do conhecimento estão agrupadas.

QUADRO 2: Práticas de gestão do conhecimento no contexto organizacional

\begin{tabular}{|c|l|}
\hline MODELO SECI & \multicolumn{1}{c|}{ PRÁTICA } \\
\hline \multirow{4}{*}{ Socialização } & Rotação dos funcionários por todas as áreas. \\
\cline { 2 - 2 } & Campings de troca de ideias ou de tormenta de ideias. \\
\cline { 2 - 2 } & Projeto de cooperação através da diretoria. \\
\cline { 2 - 2 } Internalização & Uso de mentores e aprendizes para transferir o conhecimento. \\
\cline { 2 - 2 } & Aprendizagem por observação. \\
\cline { 2 - 2 } & Aprender fazendo. \\
\cline { 2 - 2 } & Formação no posto de trabalho. \\
\cline { 2 - 2 } & Reuniões face-a-face. \\
\hline \multirow{2}{*}{ Combinação } & $\begin{array}{l}\text { Repositórios de informação, melhores práticas e lições } \\
\text { aprendidas. }\end{array}$ \\
\cline { 2 - 2 } & Acesso a dados baseados na web. \\
\cline { 2 - 2 } & Páginas web (intranet e Internet). \\
\cline { 2 - 2 } & Base de dados. \\
\hline Externalização & Captura e transferência de conhecimento de especialistas. \\
\hline
\end{tabular}




\begin{tabular}{|l|l|}
\hline MODELO SECI & \multicolumn{1}{c|}{ PRÁTICA } \\
\hline \multirow{4}{*}{ Grupos de chat/grupos de discussão baseado na web. } \\
\cline { 2 - 2 } & Sistema de apoio à tomada de decisão. \\
\cline { 2 - 2 } & Grupware e outras ferramentas de colaboração em equipe. \\
\cline { 2 - 2 } & Modelos baseados em analogias e metáforas. \\
\cline { 2 - 2 } & Conselhos (habilidades, "páginas amarelas"). \\
\cline { 2 - 2 } & $\begin{array}{l}\text { Sistema de resolução de problemas baseados em tecnologias } \\
\text { como raciocínio baseado em casos }\end{array}$ \\
\hline
\end{tabular}

Fonte: Nonaka e Takeuchi (1997)

Segundo Batista (2004) as práticas de gestão do conhecimento são definidas como práticas de gestão organizacional voltadas para a produção, retenção, disseminação, compartilhamento e aplicação do conhecimento dentro das organizações, assim como na relação com o mundo exterior. Isto envolve a captura, absorção e retroalimentação de todo o conhecimento que possa promover o desenvolvimento organizacional. Para tanto, as práticas de gestão do conhecimento deverão estar alinhadas a missão, a visão de futuro e as estratégias organizacionais.

\section{Desempenho organizacional}

Slater, Olson e Hult (2010) definem o desempenho organizacional como o atingir ou a superação dos objetivos estabelecidos pela organização, podendo ser avaliado por aspectos financeiros, de mercado e inovação. O desempenho de uma organização refere-se aos resultados por ela alcançados em certo período, que são avaliados por meio de parâmetros quantificáveis denominados indicadores (FERNANDES; FLEURY; MILLS, 2006).

Segundo Guimarães (1998) um indicador constitui uma variável mensurável, sua função é indicar o estágio de desenvolvimento desejável para a organização ou parte dela. Ao indicador é associado o conceito de meta, que designa um padrão ou referência a ser alcançada.

Historicamente, o processo de avaliação do desempenho organizacional esteve atrelado à utilização de relatórios financeiros, que expressavam os resultados de uma organização a partir de medidas como lucratividade, rentabilidade de produtos e retorno sobre o patrimônio. Tais medidas financeiras quando utilizadas isoladamente são inadequadas para orientar e avaliar a trajetória organizacional em ambientes competitivos.

Buscando aprimorar os mecanismos de gestão do desempenho, Kaplan e Norton (1997) desenvolveram o Balanced Scorecard (BSC), um sistema gerencial que pretende equilibrar a definição de indicadores e metas em diferentes perspectivas ou dimensões, conforme ilustrado na Figura 1.

Figura 1: Perspectivas do BSC 


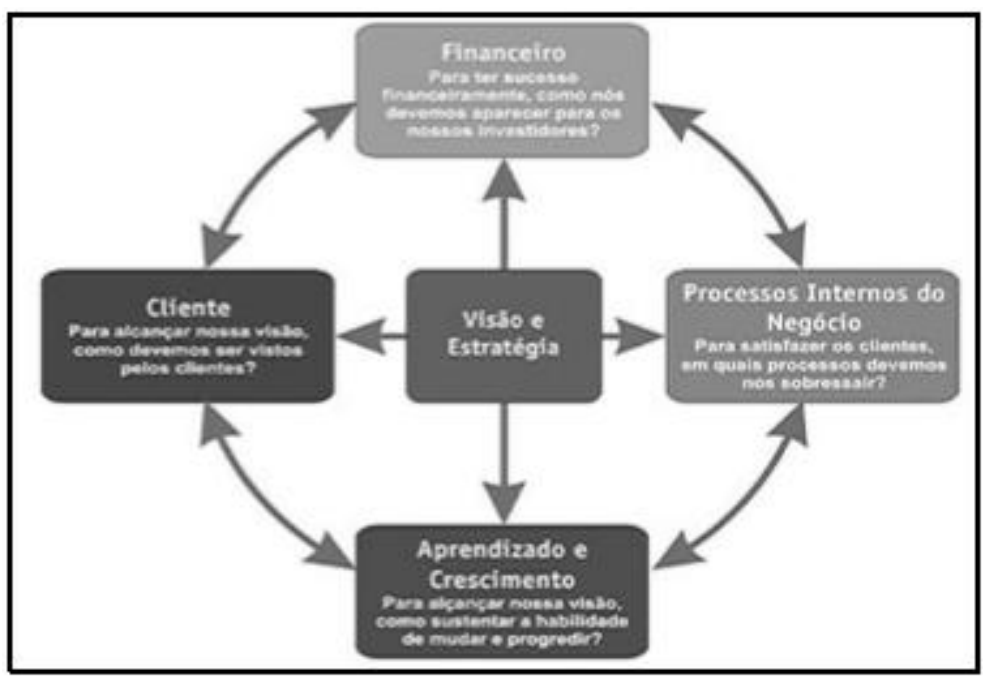

Fonte: Kaplan e Norton (1997)

Como a estratégia organizacional representa um conjunto de hipóteses sobre relações de causa e efeito, o BSC assume o pressuposto de que os objetivos e respectivos indicadores em cada perspectiva de desempenho exercem influência uns sobre os outros, de tal forma que a consecução de metas na perspectiva aprendizagem e crescimento, por exemplo, teria efeito positivo sobre os resultados associados a processos internos, e assim por diante (KAPLAN e NORTON, 1997).

\section{Modelos empíricos de gestão do conhecimento e desempenho organizacional}

Conceitualmente pode-se entender um modelo como uma estruturação simplificada da realidade que apresenta supostamente características ou correlação de forma generalizada (CHORLEY; HAGGGETT, 1975). Para Sayão (2001) os modelos são representações simplificadas e inteligíveis do mundo, que permitem vislumbrar características essenciais de um domínio ou campo de estudo, mas ainda assim, são passíveis de modificação e atualização constante para seu próprio aperfeiçoamento.

$\mathrm{Na}$ revisão sistemática realizada, não foram encontrados estudos que abordassem a aplicação empírica de modelos de gestão do conhecimento e desempenho organizacional em empresas brasileiras, por se tratar de um tema pouco abordado no país. Dentre os estudos internacionais obtidos, foram identificados os seguintes modelos (Quadro 3):

QUADRO 3: Práticas de gestão do conhecimento no contexto organizacional

\begin{tabular}{|c|}
\hline MODELOS \\
\hline Lee e Choi (2003) \\
\hline Fugate, Stank e Mentzer (2009) \\
\hline Zack, Mckeen e Singh (2009) \\
\hline Mills e Smith (2011) \\
\hline
\end{tabular}


Após a avaliação dos modelos, a pesquisa optou pelo modelo de $\mathrm{Wu}$ e Chen (2014) por abranger a gestão do conhecimento em sua completude, além de avaliar as relações da mesma com o desempenho organizacional. Outra justificativa para a seleção deste modelo é que este se adequa às empresas de base tecnológica e aos processos de negócio.

A técnica utilizada para validar o modelo foi a de mínimos quadrados parciais, que é uma técnica de modelagem de equações estruturais. As variáveis no modelo de pesquisa foram formuladas como construções de formação de segunda ordem, que foram ainda mais determinados a partir da combinação de seus indicadores formativos de primeira ordem.

O modelo de mensuração de segunda ordem foi construído para avaliar a validade convergente e discriminante da escala. A estrutura hierárquica BSC também fornece um quadro teórico abrangente para definir a relação nomológica desse modelo, segundo Wu e Chen (2014).

O modelo de Wu e Chen (2014) é ilustrado na Figura 2.

Figura 2: Modelo de gestão do conhecimento e desempenho organizacional de Wu e Chen

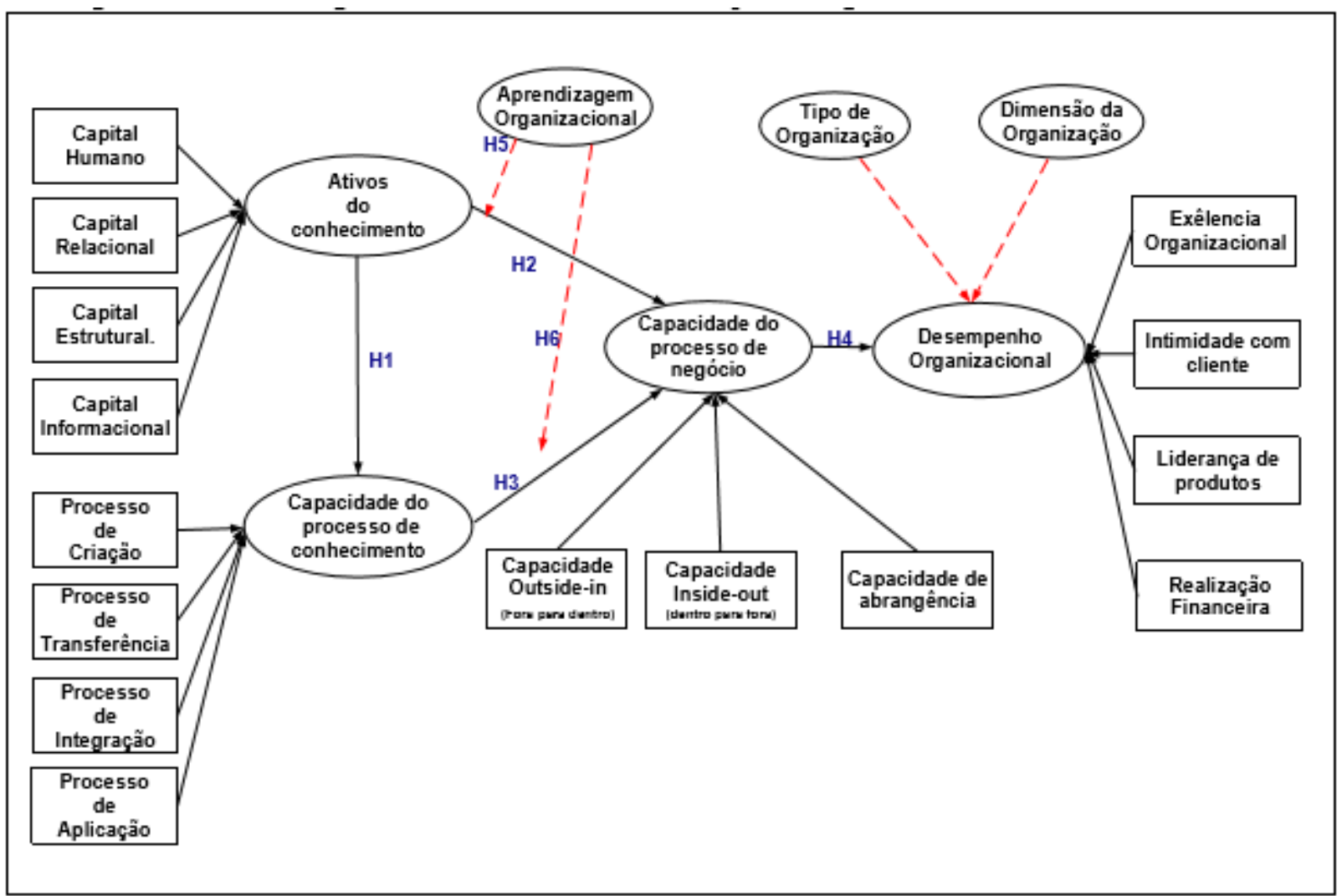

Fonte: Wu e Chen (2014)

Por meio do modelo proposto por Wu e Chen (2014), as seguintes hipóteses foram formuladas: 
- H1: Mais ativos de conhecimento levam a maior capacidade de processo de conhecimento;

- H2: Mais ativos de conhecimento levam a recursos de processos de negócios mais elevados;

- H3: Recursos de processos de conhecimento superior levam a uma maior capacidade do processo de negócio;

- H4: Recursos de processos de negócios mais elevados levar a um desempenho organizacional superior;

- H5: Aprendizado organizacional superior leva a um maior efeito moderador sobre a relação entre os ativos de conhecimento e capacidades de processos de negócios;

- H6: Aprendizado organizacional superior leva a um maior efeito moderador sobre a relação entre as capacidades de conhecimento e processos de negócios

\section{Empresas de base tecnológica e suas caracterizações}

As Empresas de Base Tecnológica são participantes ativas das mudanças tecnológicas e são capazes de apoiar o desenvolvimento socioeconômico de um país. O centro das EBT está na criação de caminhos que propiciem a transferência de tecnologias que sejam orientadas ao mercado e tem-se como resultado a comercializem de bens de consumo inovadores baseados no conhecimento científico e tecnológico (SARQUIS et al., 2015).

Pinho et al. (2005) salientam que as EBTs, principalmente as brasileiras, se concentram na realização de esforços tecnológicos significativos para a fabricação de produtos "incrementais" provenientes da imitação, adaptação ou engenharia reversa. Esse tipo de fabricação permite a construção de produtos inovadores que são capazes de substituir aqueles importados.

As EBTs podem se beneficiar de oportunidades advindas da inovação e, por meio de rotinas menos rígidas e mais flexíveis, apresentam uma elevada expectativa de crescimento e escalabilidade (SANTOS; PINHO, 2010; HYYTINEN; PAJARINEN; ROUVINEN, 2015).

Ao se estudar as Empresas de Base Tecnológica brasileiras, Santos e Pinho (2010) avaliaram a existência de uma taxa de crescimento maior que em outras organizações, o que faz das EBTs uma promessa mercadológica.

Por meio dessas empresas, é possível valorizar tecnologias e procedimentos disponíveis nas universidades, instituições, centros de pesquisas e, até mesmo, nas empresas, motivo esse que a torna fundamental no desempenho econômico e social.

Vale ressaltar que, mesmo com todas as vantagens proporcionadas pelas EBTs, esse tipo de empresa é caracterizado por um grau elevado de risco e pela necessidade de investimento, o que impacta diretamente o 
seu desenvolvimento e desempenho (BOCKEN, 2015). São exemplos de ramos de atuação dessas empresas: informática, eletrônica, mecatrônica, produção de materiais, instrumentalização, automação, química, entre outros.

\section{Procedimentos metodológicos}

A pesquisa é descritiva e se caracteriza, preponderantemente, por uma abordagem quantitativa. A análise quantitativa é apropriada para medir tanto opiniões, atitudes e preferências como comportamentos e a pesquisa descritiva expõe características de determinada população ou de determinado fenômeno (MORESI, 2003).

A coleta de dados foi realizada por meio de envio de questionário online (e-survey) a colaboradores de empresas de base tecnológica, independente do cargo que ocupavam. A escolha se deu de forma aleatória, por meio de contatos realizados via redes sociais, não sendo possível mensurar o universo da pesquisa. Ao total, foram obtidas 275 respostas, das quais 57 foram descartadas por apresentarem mais de $10 \%$ das respostas inconsistentes. Ao final, tem-se 212 respostas válidas para análise.

Para análise utilizou-se a Modelagem de Equações Estruturais, utilizando a abordagem PLS. A abordagem PLS (Partial Least Squares) oferece uma alternativa a abordagem tradicional baseada na estrutura de covariância, CB-SEM (Covariance-based Structural Equation Modeling techniques)... Para verificar a qualidade dos ajustes foi utilizado o R2, para comparar os índices com as variáveis qualitativas nominais foi utilizado o teste de Mann-Whitney e Kruskal Wallis e para comparar os índices com as variáveis quantitativas e qualitativas ordinais foi utilizada a Correlação de Spearman (MONECKE; LEISCH, 2012; HENSELER, 2012; MONECKE; LEISCH, 2012).

\section{Resultados da pesquisa}

$\mathrm{Na}$ análise dos outliers (que são observações que apresentam um padrão de resposta diferente das demais), não foram encontrados valores fora do intervalo da escala de sua respectiva variável. Ja os outliers multivariados foram diagnosticados com base na medida $D^{2}$ de Mahalanobis.

De acordo com Hair, et al. (2009), tal medida verifica a posição de cada observação comparada com o centro de todas as observações em um conjunto de variáveis, sendo que, ao final, é realizado um teste quiquadrado. Os indivíduos que apresentam uma significância da medida inferior a 0,001 foram considerados outliers multivariados. Com base neste método, foram encontrados 7 indivíduos atípicos.

Para verificar a linearidade dos dados, foram analisadas as correlações das variáveis par a par, uma vez que um coeficiente de correlação significativo ao nível de $5 \%$ é indicativo da existência de linearidade. Através da matriz de correlação de Spearman (HOLLANDER; 
WOLFE, 1999), foram observadas 944 de 946 relações significativas ao nível de $5 \%$, o que representa aproximadamente $99,78 \%$ das correlações possíveis.

Os constructos "Ativos do Conhecimento", "Capacidade do Processo de Conhecimento", "Capacidade do Processo de Negociação" e "Desempenho Organizacional" foram considerados como constructos de segunda ordem, ou seja, não eram formados diretamente pelos itens (perguntas), mas por outras variáveis latentes (indicadores). Para tratar essa característica da estrutura de mensuração, foi utilizada a abordagem "TwoStep" (SANCHEZ, 2013). Dessa forma, primeiramente foram computados os escores das variáveis latentes de primeira ordem, utilizando a Análise Fatorial Exploratória (AFE) com o método de extração dos componentes principais e rotação promax (MINGOTI, 2007).

Para os constructos de primeira ordem, a Análise Fatorial Exploratória tem o objetivo de verificar a necessidade de excluir algum item (pergunta) dos constructos que não esteja contribuindo com a formação dos índices. Segundo Hair et al (2009), itens com cargas fatoriais menores que 0,50 devem ser eliminados dos constructos, pois, não contribuem de forma relevante para formação da variável latente, prejudicando assim o alcance das suposições básicas para validade e a qualidade dos indicadores criados para representar o conceito de interesse.

Para analisar a qualidade e validade dos constructos de primeira ordem foi verificada a dimensionalidade, confiabilidade e validade convergente. Para verificar a validade convergente foi utilizado o critério proposto por Fornell e Larcker (1981), que indica a validação convergente quando a variância média extraída (AVE) for superior a $50 \%$, ou $40 \%$ no caso de pesquisas exploratórias (NUNNALY et al.,1994; HENSELER et al., 2009).

Para mensurar a confiabilidade foi utilizado o Alfa de Cronbach e a Confiabilidade Composta (CHIN, et al., 1998). Segundo Tenenhaus et al. (2005), os indicadores Alfa de Cronbach (AC) e Confiabilidade Composta (CC) devem ser maiores que 0,70 para uma indicação de confiabilidade do constructo, sendo que em pesquisas exploratórias valores acima de 0,60 também são aceitos.

Para verificar a dimensionalidade dos constructos foi utilizado o critério de Kaiser (1958), que retorna o número de fatores que devem ser retidos na Análise Fatorial Exploratória, ou seja, a quantidade de dimensões do constructo. A adequação da amostra para utilização da Análise Fatorial Exploratória foi medida através do indicador KMO que indica a proporção da variância dos dados que pode ser considerada comum a todas as variáveis. É uma medida que varia de 0,0 a 1,0 , sendo que quanto mais próximo de 1,0 (unidade) mais apropriada será a amostra à aplicação da análise fatorial. É adequado aplicar a Análise Fatorial Exploratória ao conjunto de variáveis quando o KMO for maior que 0,50 . 
A Tabela 1 apresenta os resultados para a validade e qualidade dos constructos. Logo, pode-se destacar que:

- Todos os constructos apresentaram validação convergente $($ AVE $>0,40)$;

- Todos os constructos apresentaram Alfa de Cronbach (AC) e Confiabilidade Composta (CC) acima de 0,60. Ou seja, todos apresentaram os níveis exigidos de confiabilidade;

- Em todos os constructos o ajuste da Análise Fatorial Exploratoria foi adequado, uma vez que todos os KMO foram maiores ou iguais a 0,50.

TABELA 1: Confiabilidade, validade convergente e dimensionalidade dos indicadores dos constructos de segunda ordem.

\begin{tabular}{|c|c|c|c|c|c|c|c|}
\hline \multicolumn{2}{|c|}{ Constructos } & $\begin{array}{c}\text { Iten } \\
\mathbf{S}\end{array}$ & AVE & AC & CC & $\begin{array}{c}\text { KM } \\
\mathbf{0}\end{array}$ & $\begin{array}{l}\mathbf{D i} \\
\mathbf{m}\end{array}$ \\
\hline $\begin{array}{l}\text { Ativos do } \\
\text { Conhecimento }\end{array}$ & $\begin{array}{l}\text { Capital Humano } \\
\text { Capital Relacional } \\
\text { Capital Estrutural } \\
\text { Capital Informacional }\end{array}$ & $\begin{array}{l}2 \\
3 \\
3 \\
3\end{array}$ & $\begin{array}{c}0,8 \\
5 \\
0,7 \\
8 \\
0,7 \\
0 \\
0,8 \\
0\end{array}$ & $\begin{array}{c}0,8 \\
2 \\
0,8 \\
6 \\
0,7 \\
9 \\
0,8 \\
7\end{array}$ & $\begin{array}{c}0,8 \\
6 \\
0,8 \\
6 \\
0,8 \\
1 \\
0,8 \\
7\end{array}$ & $\begin{array}{l}0,73 \\
0,67 \\
0,72\end{array}$ & 1 \\
\hline $\begin{array}{l}\text { Capacidade do } \\
\text { Processo de } \\
\text { Conhecimento }\end{array}$ & $\begin{array}{c}\text { Processo de Criação } \\
\text { Processo de Transferência } \\
\text { Processo de Integração } \\
\text { Processo de Aplicação }\end{array}$ & $\begin{array}{l}3 \\
3 \\
3 \\
3\end{array}$ & $\begin{array}{c}0,6 \\
8 \\
0,8 \\
3 \\
0,8 \\
1 \\
0,7 \\
5\end{array}$ & $\begin{array}{c}0,7 \\
5 \\
0,9 \\
0 \\
0,8 \\
8 \\
0,8 \\
3\end{array}$ & $\begin{array}{c}0,7 \\
9 \\
0,8 \\
9 \\
0,8 \\
7 \\
0,8 \\
4\end{array}$ & $\begin{array}{l}0,61 \\
0,74 \\
0,72 \\
0,70\end{array}$ & 1 \\
\hline $\begin{array}{l}\text { Capacidade do } \\
\text { Processo de } \\
\text { Conhecimento }\end{array}$ & $\begin{array}{l}\text { Capacidade de Outside-in } \\
\text { Capacidade de Inside-out } \\
\text { Capacidade de Abrangência }\end{array}$ & $\begin{array}{l}4 \\
4 \\
4\end{array}$ & $\begin{array}{c}0,7 \\
3 \\
0,6 \\
8 \\
0,7 \\
4\end{array}$ & $\begin{array}{c}0,8 \\
8 \\
0,8 \\
4 \\
0,8 \\
8\end{array}$ & $\begin{array}{c}0,8 \\
6 \\
0,8 \\
4 \\
0,8 \\
7\end{array}$ & $\begin{array}{l}0,82 \\
0,80 \\
0,83\end{array}$ & 1 \\
\hline $\begin{array}{l}\text { Capacidade do } \\
\text { Processo de Negócio }\end{array}$ & $\begin{array}{c}\text { Excelência Operacional } \\
\text { Intimidade com o Cliente } \\
\text { Liderança de Produtos } \\
\text { Realização Financeira }\end{array}$ & $\begin{array}{l}2 \\
2 \\
2 \\
2\end{array}$ & $\begin{array}{c}0,8 \\
7 \\
0,9 \\
3 \\
0,8 \\
3 \\
0,8 \\
9\end{array}$ & $\begin{array}{c}0,8 \\
5 \\
0,9 \\
3 \\
0,8 \\
0 \\
0,8 \\
8\end{array}$ & $\begin{array}{c}0,8 \\
8 \\
0,9 \\
3 \\
0,8 \\
4 \\
0,9 \\
0\end{array}$ & $\begin{array}{l}0,50 \\
0,50 \\
0,50 \\
0,50\end{array}$ & 1 \\
\hline
\end{tabular}

Fonte: Dados da pesquisa.

\section{Modelagem de equações estruturais}

Embora os constructos de primeira ordem sejam reflexivos, os constructos de segunda ordem são formativos. Dessa forma, os 
constructos de primeira ordem são as causas de seus respectivos constructos de segunda ordem, enquanto que os itens (perguntas) são os reflexos de seus respectivos constructos de primeira ordem.

A validação de um modelo de mensuração formativo requer diferentes abordagens do modelo reflexivo. A avaliação convencional de validação e confiabilidade dos constructos não deve ser aplicada nos modelos formativos (BOLLEN, 1989). Portanto, para avaliar o modelo de mensuração formativo, foi verificado se os pesos eram significativos ou maiores que 0,20 e se as cargas fatoriais eram maiores que 0,6 (LOHMOLLER, 1989; CHIN, 1998).

Uma vez que houver pesos não significativos e cargas fatoriais baixas, inexiste o suporte empírico para manter o indicador no modelo (CENFETELLI; BASSELLIER, 2009). Também foi avaliado se o Fator de Inflação da Variância (VIF) era maior que 5, evitando assim problemas de multicolinearidade. Também foi identificado que as correlações entre os constructos eram menores que 0,85, de forma a indicar que cada constructo era suficientemente diferente um do outro.

A Tabela 2 apresenta as cargas fatoriais, os pesos com seus respectivos intervalos de confiança e valores-p e o Fator de Inflação da Variância (VIF).

TABELA 2: Modelo de Mensuração.

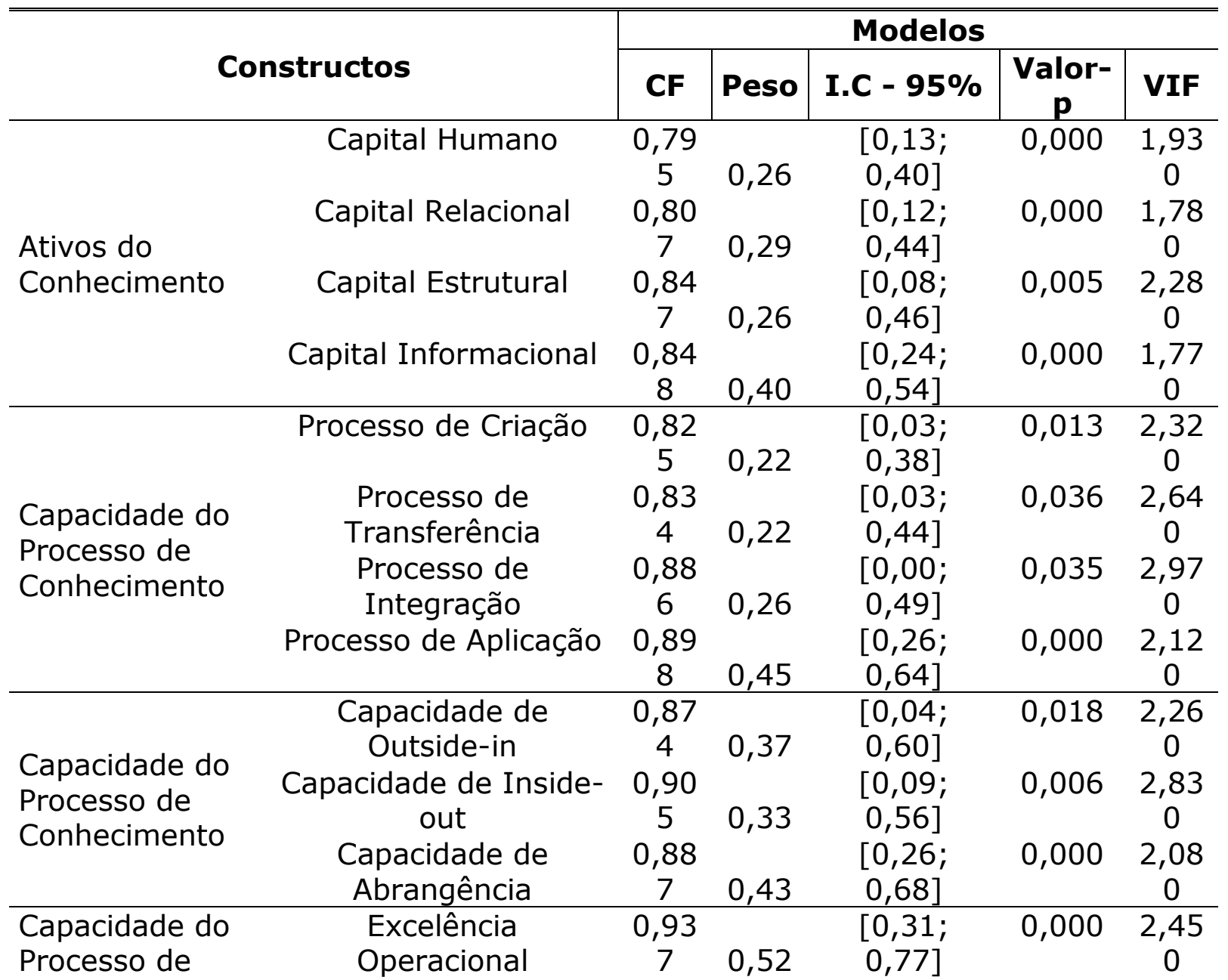


Negócio

\begin{tabular}{c|c|c|c|c|c}
\multirow{2}{*}{ Constructos } & \multicolumn{5}{c}{ Modelos } \\
\cline { 2 - 6 } & CF & Peso & I.C - 95\% & $\begin{array}{c}\text { Valor- } \\
\mathbf{p}\end{array}$ & VIF \\
\hline Intimidade com o & 0,84 & & {$[-0,05 ;$} & 0,012 & 2,72 \\
Cliente & 0 & 0,17 & $0,36]$ & & 0 \\
Liderança de Produtos & 0,85 & & {$[-0,01 ;$} & 0,049 & 2,71 \\
& 6 & 0,24 & $0,47]$ & & 0 \\
Realização Financeira & 0,76 & & {$[0,03 ;$} & 0,016 & 1,75 \\
& 1 & 0,21 & $0,36]$ & & 0 \\
\hline
\end{tabular}

Fonte: Dados da pesquisa.

O modelo de mensuração e o modelo estrutural foram realizados utilizando o método PLS. Modelos de Equações Estruturais são populares, sendo a abordagem PLS uma alternativa a abordagem tradicional baseada na covariância. A abordagem PLS, tem sido referida como uma técnica de modelagem suave com o mínimo de demanda, ao se considerar as escalas de medidas, o tamanho amostral e distribuições residuais (MONECKE; LEISCH, 2012).

Para verificar o efeito moderador da Aprendizagem Organizacional sobre a relação entre Ativos do Conhecimento e Capacidade do Processo do Negócio e também entre Capacidade do Processo de Conhecimento e Capacidade do Processo do Negócio foi utilizada a Abordagem Produto Indicador (SANCHEZ, 2013). A Abordagem do Produto Indicador cria uma nova variável latente produto da variável exógena e do efeito moderador, sendo esse produto uma nova variável no modelo estrutural. Um efeito moderador é causado por uma variável que influencia na força ou na direção da relação entre uma variável independente e uma variável dependente (BARON E KENNY, 1986). Para verificar a qualidade do ajuste foi utilizado o R2. O R2 representa em uma escala de 0 a 100 o quanto os constructos independentes explicam os dependentes, sendo que quanto mais próximo de $100 \%$ melhor.

Segundo os resultados apresentados na Tabela 3 e na Figura 3 temse que:

- Houve influência significativa (Valor- $p=0,000$ ) e positiva $(\beta=0,716[0,65 ; 0,80])$ dos Ativos de Conhecimento sobre a Capacidade do Processo de Conhecimento.

- Houve influência significativa (Valor- $p=0,000$ ) e positiva $(\beta=0,359[0,21 ; 0,52])$ dos Ativos de Conhecimento sobre a Capacidade do Processo de Negócio. Sendo assim, quanto maiores os Ativos de Conhecimento, maior tende a ser a Capacidade do Processo de Negócio;

- Houve influência significativa (Valor- $p=0,000$ ) e positiva $(\beta=0,550[0,38 ; 0,68])$ da Capacidade do processo de conhecimento sobre a Capacidade do Processo de Negócio. Sendo assim, quanto maior a Capacidade do processo de conhecimento, maior tende a ser a Capacidade do Processo de Negócio; 
O impacto das práticas de gestão do conhecimento no desempenho organizacional: um estudo em empresas de base tecnológica
Fabricio Ziviani; Erik Paixão Amarante; Renata de Souza França; Paulo Isnard; Eric de Paula Ferreira

- Houve influência significativa (Valor- $p=0,000$ ) e positiva $(\beta=0,744[0,66 ; 0,82])$ da Capacidade do processo de negócio sobre o Desempenho Organizacional. Sendo assim, quanto maior a Capacidade do processo de negócio, maior tende a ser o Desempenho Organizacional;

TABELA 3: Modelo Estrutural.

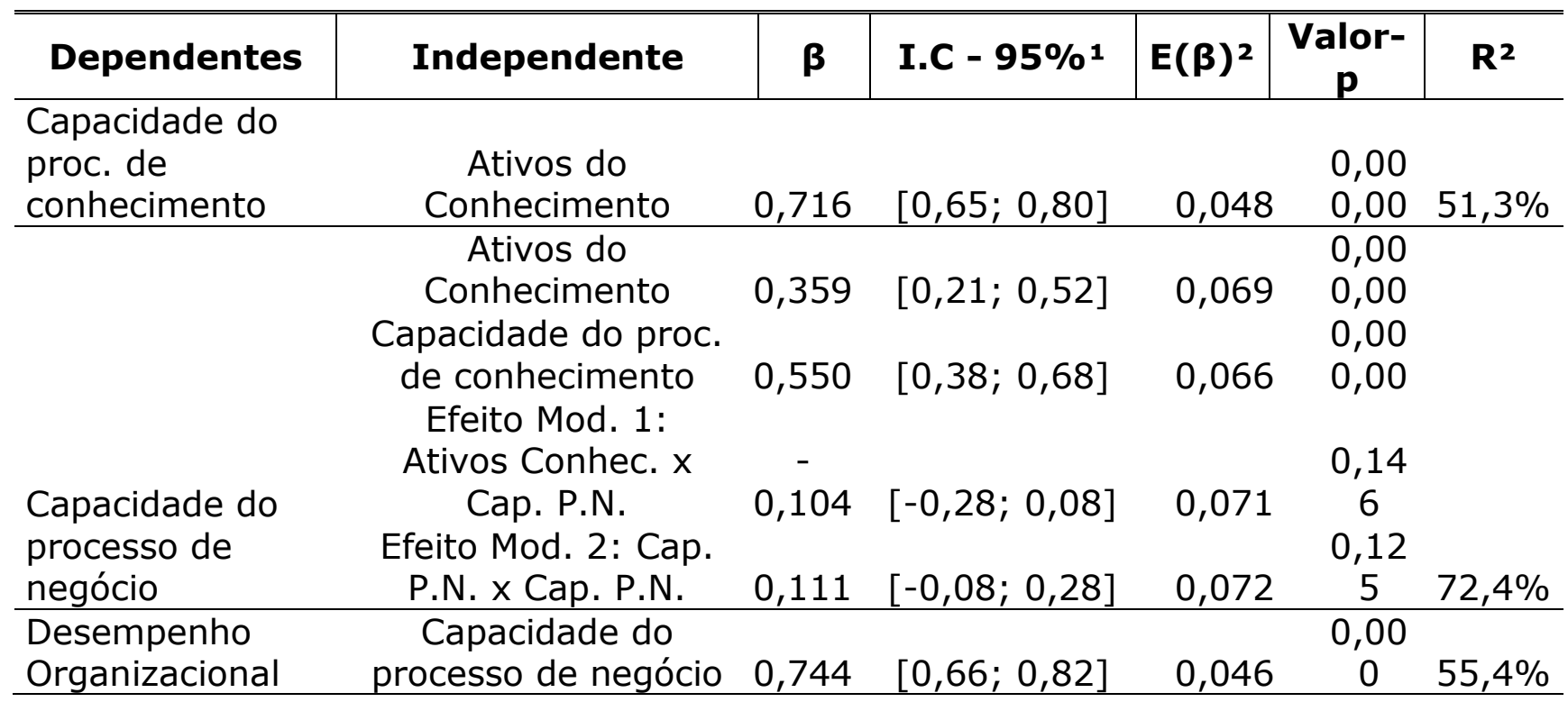

Fonte: Dados da pesquisa.

Figura 3: Ilustração do Modelo Estrutural

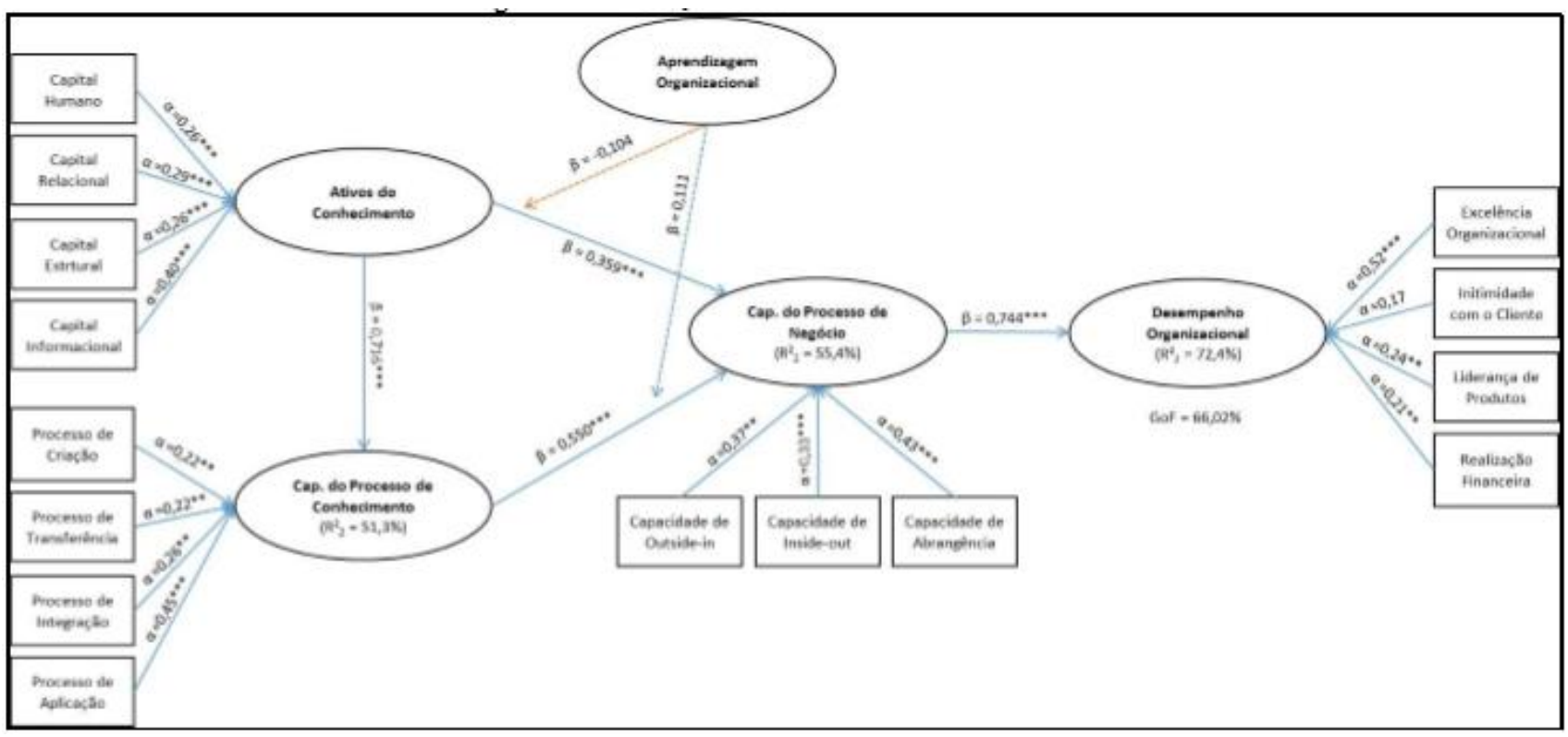

Fonte: Dados da Pesquisa

Analisando os resultados obtidos com a pesquisa, pode-se constatar que as hipóteses $\mathrm{H} 1, \mathrm{H} 2, \mathrm{H} 3$ e $\mathrm{H} 4$ foram confirmadas e as hipóteses $\mathrm{H} 5$ e H6 não foram confirmadas, conforme descrito no Quadro 3:

QUADRO 3: Atributos da Qualidade da Informação 


\begin{tabular}{|l|l|}
\hline \multicolumn{1}{|c|}{ HIPÓTESES } & RESULTADO \\
\hline $\begin{array}{l}\text { H1: Mais ativos de conhecimento levam a maior capacidade de } \\
\text { processo de conhecimento }\end{array}$ & Confirmado \\
\hline $\begin{array}{l}\text { H2: Mais ativos de conhecimento levam a recursos de } \\
\text { processos de negócios mais elevados }\end{array}$ & Confirmado \\
\hline $\begin{array}{l}\text { H3: Recursos de processos de conhecimento superior levam a } \\
\text { uma maior capacidade do processo de negócio }\end{array}$ & Confirmado \\
\hline $\begin{array}{l}\text { H4: Recursos de processos de negócios mais elevados levar a } \\
\text { um desempenho organizacional superior. }\end{array}$ & Confirmado \\
\hline $\begin{array}{l}\text { H5: Aprendizado organizacional Superior leva a um maior efeito } \\
\text { moderador sobre a relação entre os ativos de conhecimento e } \\
\text { capacidades de processos de negócios. }\end{array}$ & $\begin{array}{l}\text { Não } \\
\text { Confirmado }\end{array}$ \\
\hline $\begin{array}{l}\text { H6: Aprendizado organizacional Superior leva a um maior efeito } \\
\text { moderador sobre a relação entre as capacidades de } \\
\text { conhecimento e processos de negócios. }\end{array}$ & $\begin{array}{l}\text { Não } \\
\text { Confirmado }\end{array}$ \\
\hline
\end{tabular}

Fonte: Dados da Pesquisa

\section{Considerações finais}

O trabalho teve como objetivo analisar o impacto das práticas de gestão do conhecimento no desempenho organizacional, através do modelo de gestão do conhecimento e desempenho organizacional de Wu e Chen (2014), utilizando como base de pesquisa empresas de base tecnológicas.

Para atingir o objetivo proposto, foram estabelecidos os seguintes objetivos estratégicos:

- Traçar uma revisão sistemática de literatura a partir de modelos de gestão do conhecimento e desempenho organizacional em base de dados com descritos préestabelecidos;

- Testar e validar as hipóteses do modelo de WU e Chen (2014) em empresas de base tecnologicas brasileiras;

A escolha por empresas de base tecnológica deu-se pelas particularidades desse tipo de organização. Além dessas empresas na maioria das vezes serem compostas por estudantes universitários, recémformados ou pesquisadores de determinada área, são grandes fontes consideráveis de tecnologia.

Pode-se ressaltar que tanto o objetivo geral, como os objetivos específicos foram alcançados por essa pesquisa. Isso pode ser visto, pois:

- Foi realizada uma revisão sistemática na qual foram encontrados 42 modelos de gestão do conhecimento e desempenho organizacinal, dentre os quais foram selecionados 5 modelos por serem mais robustos e com uma maior aderência os objetivos traçados;

- Dentro os 5 modelos avaliados, a pesquisa optou pelo modelo de Wu e Chen (2014) por abranger a gestão do conhecimento em sua completude, além de avaliar as 
relações da mesma com o desempenho organizacional. Outra justificativa para a seleção deste modelo é que este se adequa a empresas de base tecnológica e aos processos de negócio;

- As hipoteses levantadas no modelo de Wu e Chen (2014) foram validadas no contexto de empresas de base tecnologicas brasileras.

Em se tratando das hipóteses definidas na pesquisa, foram alcançados os seguintes resultados:

- Houve influência significativa (Valor-p=0,000) e positiva $(\beta=0,716[0,65 ; 0,80])$ dos Ativos de Conhecimento sobre a Capacidade do Processo de Conhecimento. Sendo assim, quanto maiores os Ativos de Conhecimento, maiores tendem a ser a Capacidade do Processo de Conhecimento;

- Houve influência significativa (Valor- $p=0,000)$ e positiva $(\beta=0,359[0,21 ; 0,52])$ dos Ativos de Conhecimento sobre a Capacidade do Processo de Negócio. Sendo assim, quanto maiores os Ativos de Conhecimento, maior tende a ser a Capacidade do Processo de Negócio;

- Houve influência significativa (Valor- $p=0,000)$ e positiva $(\beta=0,550[0,38 ; 0,68])$ da Capacidade do processo de conhecimento sobre a Capacidade do Processo de Negócio. Sendo assim, quanto maior a Capacidade do processo de conhecimento, maior tende a ser a Capacidade do Processo de Negócio;

- Houve influência significativa (Valor- $p=0,000)$ e positiva $(\beta=0,744[0,66 ; 0,82])$ da Capacidade do processo de negócio sobre o Desempenho Organizacional. Sendo assim, quanto maior a Capacidade do processo de negócio, maior tendem a ser o Desempenho Organizacional;

Nota-se que dentre os construtos, o que apresenta maior influência positiva (de $74,3 \%$ ) sobre o desempenho organizacional é o processo de negócio, mas percebe-se que todos os construtos têm uma influência positiva sobre o desempenho organizacional.

A pesquisa contribuiu para semear os conceitos de gestão do conhecimento e desempenho organizacional dentro de organizações de base tecnológica de forma a apontar que, se as práticas de gestão do conhecimento forem bem definidas e trabalhadas, elas podem impactar positivamente o desempenho organizacional.

O estudo proposto se limitou a pesquisar apenas empresas de base tecnológica brasileiras. Além disso, pela forma de coleta de dados não é possível identificar o universo da pesquisa. Assim, uma sugestão de pesquisa futura seria a realização da mesma pesquisa em outros 
segmentos como indústria ou serviços em âmbito nacional, ou empresas de um determinado estado da federação.

\section{Referências}

APO. Asian Productivity Organization. Knowledge Management Tools and Techniques Manual. Tokyo, 2010.

BASKERVILLE, R.; DULIPOVICI, A. The theoretical foundations of knowledge management. Knowledge Management Research \& Practice, v. 4, n. 2, p. 83-105, 2006.

BARON, R. M.; KENNY, D. A. The moderator-mediator variable distinction in social psychological research: Conceptual, strategic, and statistical considerations. Journal of personality and social psychology, 1986.

BATISTA, F. F. O governo que aprende: gestão do conhecimento em organizações do executivo federal. Brasília: Ipea (Texto para Discussão n. 1022), 2004.

BECERRA-FERNANDEZ, I.; SABHERWAL, R. Organizational knowledge management: a contingency perspective, Journal of Management Information Systems, v. 18, p. 23-55, 2001.

BOCKEN, N. M. P. Sustainable venture capital e catalyst for sustainable start-up success? Journal of Cleaner Production, v. 108, p. 647- 658, 2015.

BOLLEN, K. A. Structural Equations with Latent Variables. New York: John Wiley \& Sons, 1989.

BRANDÃO, H. P.; GUIMARÃES, T. A. Gestão de competências e gestão de desempenho: tecnologias distintas ou instrumentos de um mesmo constructo? Revista de Administração de Empresas (RAE), São Paulo, v. 41 , n. 1, p. 8-15, 2001.

CHORLEY, Richard; Haggett, Peter. Modelos, paradigmas e a nova geografia. In: CHORLEY, Richard, HAGGETT, Peter. Modelos sócioseconômicos em geografia. Rio de janeiro : Livros Técnicos e Científicos/USP, 1975. p. 1-22.

CENFETELLI, R. T.; BASSELLIER, G. Interpretation of formative measurement in information systems research. MIS Quarterly, v. 33, n. 4, p. 698-707, 2009.

CHIN W. W.; HENSELER, J.; WANG, H. How to write up and report PLS analyses. In: ESPOSITO, V. V.; CHIN W. W.; HENSELER, J.; WANG, H. (Ed.) Handbook of Partial Least Squares. New York: Spring, 2010. p. 655690.

COOMBS, R.; HULL, R.; PELTU, M. Knowledge Management Practices for Innovation: an audit tool for improvement. International Journal of Technology Management, v. 20, n. 5-8, Jan. 2000. 
DAVENPORT, T. H. Knowledge management and the broader firm: strategy, advantage, and performance. In: LIEBOWIT, J. (Ed.). Knowledge Management Handbook. Boca Raton, CRC Press, 1999. p. 1-11.

DAVENPORT, T. H; PRUSAK, L. Conhecimento Empresarial. Rio de Janeiro: Campus, 1999.

FERNANDES, B. H.; FLEURY, M. T.; MILLS, J. Construindo o diálogo entre competência, recursos e desempenho organizacional. Revista de Administração de Empresas, São Paulo, v. 46, n.4,p.48-65, out./dez. 2006.

FORNELL, C. e LARCKER, D. F. Evaluating structural equation models with unobservable variables and measurement error. Journal of marketing research, v. 18, n. 1, p.39-50, Feb. 1981.

FUGATE, S. B; STANK, P. T; MENTZER, T. J. Linking improved knowledge management to operational and organizational performance. Journal of Operations Management 27, 2009.

GUIMARÃES, T.A. Gestão do desempenho em organizações públicas descentralizadas. In: CONGRESSO INTERNACIONAL DO CLAD, 3., 1998, Madri. Anais [...] Madri: Clad, 1998.

HAIR, J. F.; BLACK, W. C.; BABIN, B. J.; Anderson, R. E. E Tatham, R. L. Análise Multivariada de Dados. Porto Alegre: Bookman. 2009.

HENSELER, J.; RINGLE, C. M. e SINKOVICS, R. R. The use of partial least squares path modeling in international marketing. Advances in international marketing, v. 20, n. 1, 2009.

HYYTINEN, Ari; PAJARINEN, Mika; ROUVINEN, Petri. Does innovativeness reduce startup survival rates? Journal of Business Venturing, v. 30, p. 564-581, 2015.

HOLLANDER, M.; WOLFE, D. A. Nonparametric Statistical Methods. New York: John Wiley \& Sons, 1999.

KITCHENHAM, B. A. Procedures for performing systematic reviews. Keele: Department of Computer Science, Keele University; 2004. Disponível em: http://www.inf.ufsc.br/ awangenh/kitchenham.pdf. Acesso em: 18 Ago. 2014.

KAISER, H. F. The varimax criterion for analytic rotation in factor analysis. Psychometrika, v. 23, n. 3, p. 187-200, 1958.

KAPLAN, R.; NORTON, D. P. A estratégia em ação: balanced scorecard. Rio de Janeiro: Campus, 1997.

KURIAKOSE, K. K.; RAJ, B.; MURTY, S. A. V. S.; SWAMINATHAN, P. Knowledge Management Maturity Models - A Morphological Analysis. Journal of Knowledge Management Practice, v. 11, n. 3, p. 1-10, 2010.

LEE, H.; CHOI, B. Knowledge management enablers, processes, and organizational performance: an integrative view and empirical 
examination. Journal of Management Information Systems, v. 20, n. 1, p. 179-228, 2003.

LOHMOLLER, J. B. Latent variable path modelling with partial least squares. Heidelberg, Germany: Physica Verlag, 1989.

MILLS, M. A.; SMITH, A. T. Knowledge management and organizational performance: a decomposed view. Journal of Knowledge Management, v. 15, n.1, p. 156 - 171, 2011.

MINGOTI, S. A. Análise de Dados Através de Métodos de Estatística Multivariada: Uma Abordagem Aplicada. Belo Horizonte: UFMG, 2007.

MONECKE, A.; LEISCH, F. semPLS: Structural Equation Modeling Using Partial Least Squares. Journal of Statistical Software, v. 48, n. 3, p. 1-32, May 2012.

MORESI, E. A. D. (Org). Manual de Metodologia da Pesquisa. Brasília-DF: Universidade Católica de Brasília - UCB, mar., 2003.

NEELY, A.; GREGORY, M.; PLATTS, K. Performance measurement system design - A literature review and research agenda. Internacional Journal of operations \& Production Managemant, v. 15, n. 4, p. 80-116, 2005.

NONAKA, I.; TAKEUCHI, H. Criação de conhecimento na empresa: como as empresas japonesas geram a dinâmica da inovação. Rio de Janeiro: Campus, 1997.

NUNNALY, J.; BERNSTEIN, I. H. Psychometric Theory. McGraw-Hill: New York. 1994.

PINHO, M. et al. Empresas de Base Tecnológica. São Carlos: Universidade Federal de São Carlos, 2005. Relatório de Pesquisa.

PORTER, M. E. Vantagens Competitivas: criando e sustentando um desempenho superior. Rio de Janeiro: Campus, 1998.

PROBST, G.; RAUB, S.; ROMHARDT, K. Gestão do conhecimento: os elementos construtivos do sucesso. Porto Alegre: Bookman, 2002.

SABBAG, P. Y. Espirais do conhecimento: ativando indivíduos, grupos e organizações. São Paulo: Saraiva, 2007.

SANCHEZ, G. PLS Path Modeling with R. Berkeley: Trowchez Editions. 2013.

SANTOS, Daniela Tatiane dos Santos; PINHO, Marcelo. Análise do crescimento das empresas de base tecnológica no Brasil. Produção, v. 20, n. 2, p. 214-223, abr./jun. 2010.

SARQUIS, Aléssio Bessa et al. Influence factors in the innovation process technology based companies. Revista Catarinense da Ciência Contábil, Florianópolis, v. 14, n. 43, p. 38-50, set./dez. 2015.

SAYÃO, L. F. Modelos teóricos em ciência da informação: abstração e método científico. Ciência da Informação, Brasília, v. 30, n. 1, p. 82-91, jan./abr. 2001. 
SCORNAVACCA J. E.; BECKER J. L.; BARNES, S. J. Developing automated esurvey and control tools: na application in industrial mangement. Industrial mangement \& data system, v. 104, n. 3, 2004.

SLATER, S. F.; OLSON, E. M.; HULT, G. T. M. Worried about strategy implementation? Don't overlook marketing's role. Business Horizons, v. 53, n. 5, p. $469-479,2010$.

TENENHAUS, M.; VINIZI, V.; CHATELIN, Y. e LAURO, C. PLS path modeling. Computacional statistics \& Data Analysis, v. 48, n. 1, p. 159205, 2005.

TERRA, J. C. Gestão do conhecimento: o grande desafio empresarial. Rio de Janeiro: Elsevier, 2005.

WU, I. L.; CHEN, J. L. Knowledge management driven firm performance: the roles of business process capabilities and organizational learning. Journal of Knowledge Management, v. 18, n. 1, p. 185-2014, 2014.

ZACK, M.; MCKEEN, J.; SINGH, S. Knowledge management and organizational performance: an exploratory analysis. Journal of Knowledge Management, v. 13, n. 6, p. 392-409, 2009. 